\title{
Valuing Convertible Bonds Based on LSRQM Method
}

\author{
Jian Liu, ${ }^{1}$ Lizhao Yan, ${ }^{2}$ and Chaoqun $\mathrm{Ma}^{3}$ \\ ${ }^{1}$ School of Economics and Management, Changsha University of Science and Technology, Changsha 410004, China \\ ${ }^{2}$ Press, Hunan Normal University, Changsha 410081, China \\ ${ }^{3}$ Business School, Hunan University, Changsha 410082, China \\ Correspondence should be addressed to Jian Liu; ljorg@126.com
}

Received 11 March 2014; Accepted 23 May 2014; Published 24 June 2014

Academic Editor: Chuangxia Huang

Copyright (C) 2014 Jian Liu et al. This is an open access article distributed under the Creative Commons Attribution License, which permits unrestricted use, distribution, and reproduction in any medium, provided the original work is properly cited.

Convertible bonds are one of the essential financial products for corporate finance, while the pricing theory is the key problem to the theoretical research of convertible bonds. This paper demonstrates how to price convertible bonds with call and put provisions using Least-Squares Randomized Quasi-Monte Carlo (LSRQM) method. We consider the financial market with stochastic interest rates and credit risk and present a detailed description on calculating steps of convertible bonds value. The empirical results show that the model fits well the market prices of convertible bonds in China's market and the LSRQM method is effective.

\section{Introduction}

Convertible bond is a hybrid financial derivative with the properties of the bond and option which can be converted to the underlying common stocks. The outstanding US convertible bond market stood at $\$ 240$ billion in 2012 while the accumulative issuance was $\$ 404$ billion from 1980 to 2011. The pricing problem is the key to the convertible bonds research, for the prices of convertible bonds immediately influence both the profits of the investors and the financing costs of the issuers.

The convertible bonds are difficult to be priced due to embedded American-style options from the provisions, such as callability and puttability, where the ability of the issuing firm to exercise its options depends on the path of the underlying stock price. Due to the uncertainty of the optimal exercise time, the problem of American option pricing cannot be solved with analytical solution methods such as B-S formula but can be solved with numerical methods, such as binary tree method, finite difference method, and finite element method. The binary tree method introduced by Cox et al. [1] is one of the most influential numerical methods to price American options. However, it is likely to increase the computation amount and may cause the curse of dimensionality when there are multiple stochastic factors in the market model. The finite difference method also hardly disposes the situation with two or more stochastic factors. Another choice is the Monte Carlo simulation method. Ammann et al. [2] show that the relationship between the number of stochastic factors and computing time is almost linear in the Monte Carlo framework. But the normal Monte Carlo method usually only suits the European options pricing, because the optimal exercise strategy algorithm to American options is backward while the Monte Carlo method is forward, which may cause incompatibility and generate deviation.

Longstaff and Schwartz [3] present the Least-Squares Monte Carlo (LSM) approach for pricing American options by simulation. Stentoft [4] makes a detailed analysis of the LSM approach and shows that the LSM method is computationally more efficient than finite difference methods and the Binomial Model when the number of assets is high. However, the pseudorandom number generated by Monte Carlo (MC) method in the LSM approach shows aggregation so that the convergence is very slow, the simulation variance is large, and the pricing efficiency is low. For that, an alternative method, namely, the quasi-Monte Carlo (QMC) method, is widely used in pricing financial derivatives [5-9]. The basic idea of QMC method is using more uniformly distributed points instead of random points. The QMC method is applied to evaluate the expectation of a random function path generated by a stochastic process and the convergence of QMC is asymptotically better than MC. However, the convergence 
order is related to the dimension, and the efficiency reduces for high-dimensional integrals. Some researchers present a Randomized Quasi-Monte Carlo (RQMC) method which overcomes the disadvantage of QMC. This paper uses a LeastSquares Randomized Quasi-Monte Carlo (LSRQM) method, which combines the LSM approach with RQMC method, to price the convertible bond.

Furthermore, as a kind of corporate bonds, the convertible bonds with long duration may involve credit risk. There are two methods to model the credit risk. One is using the credit risk premium to describe the credit risk, so the discount rate of the bond part value in convertible bonds equals the risk-free interest rate plus the credit risk premium, and the equity value is discounted by the risk-free interest rate. Details can be found in Ammann et al. [2]. The other one is using the default probability to reflect the credit risk. The value of convertible bonds turns to be the recovery value when bonds default, details can be found in Jarrow and Turnbull [10], Hung and Wang [11], Donald and Qin [12], Liu et al. [13], and $\mathrm{Xu}$ [14]; the latter uses the no-arbitrage principle to get the probability of default, and it is simple and convenient. So we use the latter method to model the credit risk involved in convertible bonds.

This paper prices convertible bonds with call and put provisions. Firstly, we model the financial market with the stochastic interest rates and the credit risk with suitable stochastic differential equations. Then, we describe the credit risk with Jarrow and Turnbull model. Furthermore, we present detailed calculation steps of convertible bonds values with LSRQM approach. Based on the theoretic analysis, we make a numerical simulation under determinate parameters and an empirical analysis using the data from China's market.

\section{Market Model}

2.1. Stock Price. Suppose the financial market is frictionless, efficient, and continuous in the time interval $[0, T]$, which is characterized by the probability space $\left(\Omega, \mathscr{F},\left\{\mathscr{F}_{t}\right\}_{t \geq 0}, P\right)$. $\Omega$ denotes the state space, $\mathscr{F}$ is the $\sigma$-algebra of measurable events, $\left\{\mathscr{F}_{t}\right\}_{t \geq 0}$ is the $\sigma$-algebra filtration generated by a twodimensional Brownian motion $\left\{\widetilde{B}^{1}, \widetilde{B}^{2} ; t \leq T\right\}$, and $P$ is the physical probability measure. Considering a convertible bond with callable and puttable provisions, the process of the underlying stock price satisfies the stochastic differential equation given by [15]

$$
\begin{aligned}
& d \widetilde{S}_{s}=\mu\left(\widetilde{r}_{s}, \widetilde{S}_{s}, s\right) \widetilde{S}_{s} d s \\
& +\sigma_{1}\left(\widetilde{r}_{s}, \widetilde{S}_{s}, s\right) \widetilde{S}_{s} d \widetilde{B}_{s}^{1}+\sigma_{2}\left(\widetilde{r}_{s}, \widetilde{S}_{s}, s\right) \widetilde{S}_{s} d \widetilde{B}_{s}^{2}, \\
& \quad \widetilde{S}_{0}=S_{0}>0
\end{aligned}
$$

where $\widetilde{r}_{s}$ denotes the short rate at time $s, \mu$ denotes the drift, and the coefficients $\sigma_{1}$ and $\sigma_{2}$ are positive and describe the volatility of the stock price. All of the parameters are $\mathscr{F}_{t^{-}}$ adapted. As we will see, $\widetilde{B}_{s}^{2}$ represents the price fluctuation due to stock-specific randomness.
TABLE 1: Short-term interest rate models.

\begin{tabular}{lcccc}
\hline Model & $a_{0}$ & $b_{0}$ & $c(t)$ & $d(t)$ \\
\hline Vasicek & $-a_{0}$ & $b_{0}$ & 0 & $\sigma^{2}$ \\
Cox-Ingersoll-Ross & $-a_{0}$ & $b_{0}$ & $\sigma^{2}$ & 0 \\
Ho-Lee & 0 & $b_{0}$ & 0 & $\sigma^{2}$ \\
Hull-White 1 & $-a_{0}$ & $b_{0}$ & 0 & $\sigma(t)^{2}$ \\
Hull-White 2 & $-a_{0}$ & $b_{0}$ & $\sigma(t)^{2}$ & 0 \\
\hline
\end{tabular}

2.2. Interest Rate. Assume that the process of risk-free short interest rate follows the stochastic differential equation:

$$
\begin{gathered}
d \widetilde{r}_{s}=\left(a_{0}(s)+b_{0}(s) \tilde{r}_{s}\right) d s+\sqrt{c(s) \tilde{r}_{s}+d(s)} d \widetilde{B}_{s}^{1}, \\
\widetilde{r}_{0}=r_{0} .
\end{gathered}
$$

The parameters $a_{0}, b_{0}, c, d$ are the deterministic functions of time $t$. This interest rate model is a general model which can be changed into classical interest rate models such as Vasicek and CIR model as choosing different form of parameters. See details in Table 1.

Models 1 and 2 are built in the real probability space which should be transformed into the risk-neutral probability space. So, define

$$
\begin{gathered}
u_{1 t}=\frac{\left(a_{0}(t)-a(t)\right)+\left(b_{0}(t)-b(t)\right) \widetilde{r}_{t}}{\sqrt{c(t) \widetilde{r}_{t}+d(t)}}, \\
u_{2 t}=\frac{\mu_{t}-\widetilde{r}_{t}-\sigma_{1} u_{1 t}}{\sigma_{2}(t)}, \\
M_{t}=\exp \left(-\int_{0}^{t} u_{1 t} d \widetilde{B}_{t}^{1}-\int_{0}^{t} u_{2 t} d \widetilde{B}_{t}^{2}\right. \\
\left.-\frac{1}{2} \int_{0}^{t} u_{1 t}^{2} d \widetilde{B}_{t}^{1}-\frac{1}{2} \int_{0}^{t} u_{2 t}^{2} d \widetilde{B}_{t}^{2}\right) .
\end{gathered}
$$

Let $d Q(\omega)=M_{T}(\omega) d P(\omega), \omega \in \mathscr{F}_{T}^{2}$; then by Girsanov Theorem, $Q$ is the risk-neutral probability measure in $\mathscr{F}_{T}$. Define $B_{t}^{1}=\widetilde{B}_{t}^{1}+\int_{0}^{t} u_{1 s} d s, B_{t}^{2}=\widetilde{B}_{t}^{2}+\int_{0}^{t}\left(\left(\mu_{t}-\widetilde{r}_{t}-\sigma_{1} u_{1 s}\right) / \sigma_{2}\right) d s$, and then $\left(B_{t}^{1}, B_{t}^{2}\right)$ is a two-dimensional Brownian motion with respect to $Q$, and the process of risk-free interest rate satisfies the following stochastic differential equation under Q:

$$
\begin{gathered}
d r_{s}=\left(a(s)-b(s) r_{s}\right) d s+\sqrt{c(s) r_{s}+d(s)} d B_{s}^{1}, \\
r_{t}=r_{0} .
\end{gathered}
$$

The process of the stock price satisfies the following stochastic differential equation under $Q$ :

$$
\begin{gathered}
d S_{s}=r_{s} S_{s} d s+\sigma_{1}\left(r_{s}, S_{s}, s\right) S_{s} d B_{s}^{1}+\sigma_{2}\left(r_{s}, S_{s}, s\right) S_{s} d B_{s}^{2}, \\
S_{0}=S_{0}>0 .
\end{gathered}
$$

So far the pricing space is transformed into the risk-neutral probability space that ensures that the convertible bond value is no-arbitrage and unique. 
2.3. Credit Risk. We account for credit risk in the spirit of the methodology in Jarrow and Turnbull [10], where the credit risk is described by the default probability and the recovery value of the convertible bond by arbitrage-free valuation techniques. As the actual payoff in default is a complex problem, we take the recovery rate to the convertible bond in the event of default as an exogenously given constant like much literature does [10]. The recovery rate is assumed to be the same for all bonds in a given credit risk class. In this case, we can discount the cash flows both in the bond part and the equity part with the risk-free rate but not have to discount the bond part with the risk rate showing the credit spread as the usual practice. Actually, the probability of default has reflected the credit risk in the bond part, so the uncertainty is only from the fluctuation of the stock price and the risk-free rate.

As for the probability of default, many researchers have discussed its modeling. One method is to obtain the probability of default by calculating the corresponding intensity function of default which describes the default of the corporation. The intensity function model is developed by Litterman and Iben [16], Jarrow and Turnbull [10], and Duffie [17]. There are kinds of representations to the intensity function of default, such as the negative-exponential intensity model presented by Andersen and Buffum [18] and Linetsky [19], where the intensity function follows:

$$
h(S)=\frac{e}{S^{f}},
$$

for some $e>0, f>0$, and they are both constants. The negative-exponential intensity model reflects the fact that the credit risks of corporations with high stock prices are less than the corporations with low stock prices. In order to describe the positive correlation between the default risk and the fluctuation, Carr and Linetsky [20] suppose that the intensity function of default is an affine function with respect to the spot volatility of the stock price given by

$$
h(S)=g(t)+\frac{q \sigma_{S}^{2}}{S^{2(1-\alpha)}}
$$

where $g(t)$ is a nonnegative function of time and $q$ is positive integer. Duffie et al. [21] discover that the probability of default depends on the stock price index and the interest rate.

Denote the probability of default in time interval $[i-1, i]$ by $\lambda_{i}$. When the interest rate is $r_{i}$ and the stock price is $S_{i}$, the probability of default $\lambda_{t_{i}}$ at time $t_{i}$ can be expressed as

$$
\lambda_{t_{i}}=1-e^{h\left(r_{i}, S_{i}\right) \Delta t}
$$

This method is complicated for application in that it relates to the parameter estimation problem in default intensity function. If the estimation is not accurate, the reliability of default probability is directly affected.

Another method is using the no-arbitrage principle to get the probability of default, such as Jarrow and Turnbull [10]. This method is simple and convenient and can fit the existing term structure of interest rate better. The paper uses this no-arbitrage method to calculate the probability of default.
Denote the probability of default in each time interval $[i-1, i]$ by $\lambda_{t_{i}}, i=1,2, \ldots, n$, and the optimal stopping time of the convertible bond by $\tau$; let

$$
\lambda_{\tau}^{*}=-\frac{1}{\tau} \sum_{t=t_{1}}^{\tau} \ln \left(1-\lambda_{t}\right) .
$$

Then

$$
\prod_{t=t_{1}}^{\tau}\left(1-\lambda_{t}\right)=e^{-\lambda_{\tau}^{*} \cdot \tau}
$$

When $\lambda_{i}$ is small, we can obtain

$$
\lambda_{\tau}^{*} \approx \frac{\sum_{t=t_{1}}^{\tau} \lambda_{t}}{\tau} .
$$

Expression (11) means that $\lambda_{\tau}^{*}$ is the average intensity of default in the time interval $[0, \tau]$.

With the no-arbitrage principle, we get

$$
e^{-r_{\tau}^{*}}=\left[1 \cdot\left(1-\lambda_{\tau}^{*}\right)+\delta_{\tau} \cdot \lambda_{\tau}^{*}\right] e^{-R_{\tau}},
$$

where $r_{\tau}^{*}$ is the interest rate of the risky bond in the time interval $[0, \tau], R_{\tau}$ is the risk-free interest rate, and $\delta_{\tau}$ is the recovery rate of the convertible bond.

Then we have

$$
\lambda_{\tau}^{*}=\frac{1-e^{R_{\tau}-r_{\tau}^{*}}}{1-\delta_{\tau}} .
$$

So, we can firstly obtain the average intensity of default $\lambda_{\tau}^{*}$ with expression (13) and then deduce the probability of default in each time interval $\lambda_{t_{i}}, i=1,2, \ldots, n$ with expression (11). Furthermore, we assume that the recovery rate of the convertible bond is a constant $\delta$.

2.4. General Expression of Convertible Bond Value. We consider the convertible bond with call and put provisions (see Table 2). The call provision allows the issuer to demand premature redemption for the call price applicable and to announce the intention to call a certain period in advance which is known as the call notice period. The put provision allows the investor to force the issuer to prematurely repurchase the bond for a certain predefined price. Consequently, the payoff of the convertible bond depends on the optimal strategies of the investor and the issuer which relate to the holding value and the intrinsic value. In each exercise time $t_{k}$, the convertible bond has four possible strategies, hold, conversion, redemption, and put-back. If the optimal strategy is held at time $t_{k}$, the cash flow of the convertible bond is 0 or the current interest. While if the optimal strategy is conversion, redemption, or put-back, the cash flow of the convertible bond is the conversion value, redemption value, and puttable value, respectively. After careful analysis, the value of convertible bond is given by

$$
\begin{array}{r}
\max [\min (\text { holding value, call value) } \\
\text { put value, conversion value }]
\end{array}
$$


Let $\tau$ be the optimal exercise time, let $\omega$ be the path of state variables, and let $V(\omega ; \tau)$ be the value of the convertible bond at time $\tau$. Then in accordance with no-arbitrage principle, the value of the convertible bond at time 0 is given by [22]

$$
V_{0}=E^{Q}\left[e^{-\int_{0}^{\tau} r(\omega, t) d t} V(\omega ; \tau)\right],
$$

where $Q$ is the risk-neutral probability.

\section{Pricing Convertible Bond with LSRQM Method}

3.1. Calculating Steps. Suppose there are $n$ exercise times for the convertible bond for the duration $T$; that is, $0<t_{1} \leq t_{2} \leq$ $t_{3} \leq \cdots \leq t_{n}=T$, respectively. The time interval $\Delta t=T / n$. In the spirit of Tsiveriotis and Femandes [23], the value of the convertible bond can be divided into two parts. One part is the equity value which involves the value converted to stock and exercising the embedded options. The other part is the bond value which involves the present value of the convertible bond with the principal plus interest and the residual value when the convertible bond defaults [24]. Let the face value of the convertible bond be $F$, let the probability of default at the time interval $\left[t_{k}, t_{k+1}\right]$ be $\lambda_{t_{k+1}}$, let the annual interest rate be $r\left(\omega ; t_{k}\right)$, let the recovery rate be $\delta_{t_{k+1}}$, and let the recovery value be $\delta_{t_{k+1}} F$.

At any given time, the convertible bond has the possibility of default. That is to say, there are three kinds of cash flow prior to the due time, interest, equity value, and recovery value, and there are principals at the end. The interest promised in the contract is usually fixed and will be paid if the bond does not default. The equity value related to the optimal exercise time is the bond value when the bond is redeemed, sold back, and converted if the bond does not default. The bond value only has the recovery value when the bond defaults. If the bond does not default, the holding value of the convertible bond at time $t_{k+1}$ for every path can be expressed by

$$
\begin{aligned}
& h\left(\omega ; t_{k}\right) \\
& =e^{-r\left(\omega ; t_{k}\right) \cdot \Delta t} \cdot\left[\left(1-\lambda_{t_{k+1}}\right) \cdot C\left(\omega ; t_{k+1}\right)+D\left(\omega ; t_{k+1}\right)\right. \\
& \left.\cdot \prod_{t=t_{k+1}}^{t^{*}}\left(1-\lambda_{t}\right)+\lambda_{t_{k+1}} \delta_{t_{k+1}} F\right] .
\end{aligned}
$$

In general, the holding value of the convertible bond at time $t_{k+1}$ for every path can be expressed by

$$
\begin{aligned}
& h\left(\omega ; t_{k}\right) \\
& =e^{-r\left(\omega ; t_{k}\right) \cdot \Delta t} \cdot\left\{\begin{array}{l}
\prod_{t=t_{k+1}}^{t_{\tau}}\left(1-\lambda_{t}\right) \cdot C\left(\omega ; t_{k+1}\right) \\
+\prod_{t=t_{k+1}}^{t^{*}}\left(1-\lambda_{t}\right) D\left(\omega ; t_{\tau}\right)+\lambda_{t_{k+1}} \delta_{t_{k+1}} F
\end{array}\right.
\end{aligned}
$$

$$
\begin{aligned}
& +\sum_{n=k+1}^{\tau-1} e^{-\sum_{m=k+1}^{n} r\left(\omega ; t_{m}\right) \cdot \Delta t} \\
& \left.\quad \times\left(\prod_{m=k+1}^{n}\left(1-\lambda_{t_{m}}\right)\right) \lambda_{t_{n+1}} \delta_{t_{n+1}} F\right\} .
\end{aligned}
$$

$t_{\tau}$ is the optimal stopping time at which it is optimal for either the issuer or the investor to terminate the convertible bond, if the bond is not exercised before time $t_{k}$. $t^{*}$ denotes time points of paying interest, and $C\left(\omega ; t_{k+1}\right)$ denotes the time- $t_{k+1}$ discounted value of the equity part value if the bond does not default at time $t_{k+1} . D\left(\omega ; t_{\tau}\right)$ denotes the time- $t_{k+1}$ discounted value of the interest at the time interval $\left[t_{k}, t_{\tau}\right)$.

Accordingly, the value of the convertible bond at time $t_{k}$ is given by

$$
\begin{array}{r}
V\left(\omega ; t_{k}\right)=\max \left[\min \left(E H\left(\omega ; t_{k}\right), V^{\text {call }}\left(\omega ; t_{k}\right)\right),\right. \\
\left.V^{\text {con }}\left(\omega ; t_{k}\right), V^{\text {put }}\left(\omega ; t_{k}\right)\right],
\end{array}
$$

where $V^{\text {call }}\left(\omega ; t_{k}\right), V^{\text {con }}\left(\omega ; t_{k}\right), V^{\text {put }}\left(\omega ; t_{k}\right)$ denote the values of redemption, conversion, and put-back, respectively, $E H\left(\omega ; t_{k}\right)$ is the expected holding value. We can determine the optimal strategy of the convertible bond with (18).

Based on the above discussion, we get the calculation steps for the convertible bond with call and put provisions and the credit risk as follows.

(1) Simulate $M$ paths of state variables (stock and interest rate).

(2) At the last time $t_{n}$, the cash flow of the convertible bond for every path is given by

$$
C\left(\omega ; t_{n}\right)=\max \left[h\left(\omega ; t_{n}\right), V^{\text {con }}\left(\omega ; t_{n}\right)\right],
$$

where $h\left(\omega ; t_{n}\right)$ is the face value plus the interest at the maturity. Denote $t_{\tau}=t_{n}, \tau=n$.

(3) At time $t_{n-1}$, we get the holding value for every path as follows:

$$
\begin{aligned}
h\left(\omega ; t_{n-1}\right) & \\
=e^{-r\left(\omega ; t_{n-1}\right) \cdot \Delta t} \cdot\{( & \left.1-\lambda_{t_{n}}\right) \cdot\left[C\left(\omega ; t_{n}\right)+D\left(\omega ; t_{n}\right)\right] \\
& \left.+\lambda_{t_{n}} \delta_{t_{n}} F\right\} .
\end{aligned}
$$

(4) With the least square method, we regress $h\left(\omega ; t_{n-1}\right)$ on basis function of the time$t_{n-1} \quad$ state variables $\left(S\left(\omega ; t_{n-1}\right), r\left(\omega ; t_{n-1}\right)\right)$ denoted by $L_{j}\left(S\left(\omega ; t_{n-1}\right), r\left(\omega ; t_{n-1}\right)\right), \quad j=$ $1 \cdots K$, and get the regression coefficients $\left\{a_{1}\left(\omega ; t_{n-1}\right), a_{2}\left(\omega ; t_{n-1}\right), \ldots, a_{K}\left(\omega ; t_{n-1}\right)\right\}, \quad$ so the conditional expectation function is represented as a linear combination of basis functions denoted by $H\left(\omega ; t_{n-1}\right)$ as follows:

$$
H\left(\omega ; t_{n-1}\right)=\sum_{j=0}^{K} a_{j}\left(\omega ; t_{n-1}\right) \cdot L_{j}\left(S\left(\omega ; t_{n-1}\right), r\left(\omega ; t_{n-1}\right)\right) \text {. }
$$


(5) Substituting the state variables $\left(S\left(\omega ; t_{n-1}\right), r\left(\omega ; t_{n-1}\right)\right)$ into regression (21), we get the estimated expected holding value for each path $\omega$ denoted by $E \widehat{H}\left(\omega ; t_{n-1}\right)$.

(6) Comparing the $E \widehat{H}\left(\omega ; t_{n-1}\right)$ with other kinds of value, the value of convertible bond at time $t_{n-1}$ for each path $\omega$ can be expressed as

$$
\begin{array}{r}
V\left(\omega ; t_{n-1}\right)=\max \left[\min \left(E \widehat{H}\left(\omega ; t_{n-1}\right), V^{\text {call }}\left(\omega ; t_{n-1}\right)\right),\right. \\
\left.V^{\text {con }}\left(\omega ; t_{n-1}\right), V^{\text {put }}\left(\omega ; t_{n-1}\right)\right] .
\end{array}
$$

Then, the optimal strategy and the cash flow at time $t_{n-1}$ for each path are given as follows.

(a) If $V\left(\omega ; t_{n-1}\right)=E \widehat{H}\left(\omega ; t_{n-1}\right)$, the investor will keep holding the convertible bond, and the equity value at time $t_{n-1}$ is $C\left(\omega ; t_{n-1}\right)=$ $C\left(\omega ; t_{n}\right) e^{-r\left(\omega ; t_{n-1}\right) \Delta t}$.

(b) If $V\left(\omega ; t_{n-1}\right)=V^{\text {call }}\left(\omega ; t_{n-1}\right)$, the issuer will redeem the convertible bond, and the investor will obtain the call value. Then the equity value at time $t_{n-1}$ is $C\left(\omega ; t_{n-1}\right)=V^{\text {call }}\left(\omega ; t_{n-1}\right)$.

(c) If $V\left(\omega ; t_{n-1}\right)=V^{\text {con }}\left(\omega ; t_{n-1}\right)$, the investor will convert the bond into the underlying stock under the agreed conversion price and get the conversion value. Then the equity value at time $t_{n-1}$ is $C\left(\omega ; t_{n-1}\right)=V^{\text {con }}\left(\omega ; t_{n-1}\right)$.

(d) If $V\left(\omega ; t_{n-1}\right)=V^{\text {put }}\left(\omega ; t_{n-1}\right)$, the investor will sell back the bond to the issuer under the agreed price and get the put value. Then the equity value at time $t_{n-1}$ is $C\left(\omega ; t_{n-1}\right)=V^{\text {put }}\left(\omega ; t_{n-1}\right)$.

If the convertible bond is redeemed, converted, and sold back, denote $t_{\tau}=t_{n-1}, \tau=n-1$.

(7) Work backwards, and repeat step 3 to step 6 for the time $t_{n-2}, t_{n-3}, \ldots, t_{1}$. The discounted value of the cash flow calculated with the optimal strategy after time $t_{k}$ can be expressed as

$$
\begin{aligned}
h\left(\omega ; t_{k}\right)= & e^{-r\left(\omega ; t_{k}\right) \cdot \Delta t} \\
& \cdot\left\{\prod_{t=t_{k+1}}^{t_{\tau}}\left(1-\lambda_{t}\right) \cdot C\left(\omega ; t_{k+1}\right)\right. \\
& +\prod_{t=t_{k+1}}^{t^{*}}\left(1-\lambda_{t}\right) D\left(\omega ; t_{\tau}\right)+\lambda_{t_{k+1}} \delta_{t_{k+1}} F \\
& +\sum_{n=k+1}^{\tau-1} e^{-\sum_{m=k+1}^{n} r\left(\omega ; t_{m}\right) \cdot \Delta t} \\
& \left.\times\left(\prod_{m=k+1}^{n}\left(1-\lambda_{t_{m}}\right)\right) \lambda_{t_{n+1}} \delta_{t_{n+1}} F\right\} .
\end{aligned}
$$

With the least square method, we get the regression function $H\left(\omega ; t_{k}\right)$ and the estimated expected holding value $E \widehat{H}\left(\omega ; t_{k}\right)$. Then, we get the equity value of the convertible bond at each time for each path $C\left(\omega ; t_{k}\right), t_{1} \leq t_{k} \leq t_{n}=T$.

If the convertible bond is redeemed, converted, and sold back, update the optimal stopping time by $t_{\tau}=$ $t_{k}, \tau=k$

(8) Finally, the price of convertible bond at time 0 is the mean of the discounted value of time- $t_{1}$ cash flow for all $M$ paths, which can be expressed as

$$
\begin{aligned}
& V_{0}=E_{Q}\left\{e^{-r(\omega ; 0) \cdot \Delta t}\right. \\
& {\left[\prod_{t=t_{1}}^{t_{\tau}}\left(1-\lambda_{t}\right) \cdot C\left(\omega ; t_{1}\right)\right.} \\
& +\prod_{t=t_{1}}^{t^{*}}\left(1-\lambda_{t}\right) D\left(\omega ; t_{\tau}\right)+\lambda_{t_{1}} \delta_{t_{1}} F \\
& +\sum_{n=1}^{\tau-1} e^{-\sum_{m=1}^{n} r\left(\omega ; t_{m}\right) \cdot \Delta t} \\
& \left.\left.\times\left(\prod_{m=1}^{n}\left(1-\lambda_{t_{m}}\right)\right) \lambda_{t_{n+1}} \delta_{t_{n+1}} F\right] \mid \mathscr{F}_{0}\right\} \\
& =\frac{1}{M} \sum_{i=1}^{M} e^{-r\left(\omega_{i} ; 0\right) \cdot \Delta t} \\
& \cdot\left[\prod_{t=t_{1}}^{t_{\tau}\left(\omega_{i}\right)}\left(1-\lambda_{t}\right) \cdot C\left(\omega_{i} ; t_{1}\right)\right. \\
& +\prod_{t=t_{1}}^{t^{*}\left(\omega_{i}\right)}\left(1-\lambda_{t}\right) D\left(\omega_{i} ; t_{\tau}\right)+\lambda_{t_{1}} \delta_{t_{1}} F \\
& +\sum_{n=1}^{\tau\left(\omega_{i}\right)-1} e^{-\sum_{m=1}^{n} r\left(\omega ; t_{m}\right) \cdot \Delta t} \\
& \left.\times\left(\prod_{m=1}^{n}\left(1-\lambda_{t_{m}}\right)\right) \lambda_{t_{n+1}} \delta_{t_{n+1}} F\right] .
\end{aligned}
$$

3.2. RQMC Method. For the numerical method of the convertible bond pricing, an important problem is to simulate the path of state variables. The distribution of state variables is generally related to a normal distribution, so the simulation needs to generate the random numbers of normal distribution which is generated by the random numbers of uniform distribution at the interval $[0,1]$. Therefore, the simulation of random numbers with uniform distribution at the interval $[0,1]$ plays an important role in simulation. The MC method used in the LSM approach employs the pseudorandom numbers which show aggregation and make the convergence too slow. QMC method uses low-discrepancy sequences which generate more uniformly distributed points in order 
to have high accuracy. The main low-discrepancy sequences are Halton sequences, Faure sequences, Sobol sequences, and so on. Halton sequences are sensitive to the dimensions, while Faure sequences and Sobol sequences are not, so the latter two are employed in the high-dimensional situation.

Based on the Koksma-Hlawka inequality, the asymptotic convergence order of QMC method is $O\left((\log N)^{d} / N\right)$, which is generally better than the $O(1 / \sqrt{N})$ of MC method. However, $(\log N)^{d} / N$ may be smaller than $1 / \sqrt{N}$ when the dimension is high enough. In order to improve the QMC method in the high-dimensional situation, dimension reduction techniques are specially designed, such as the linear transformation method, the ordinary Brownian bridge $[25,26]$, and the principal component analysis (PCA). The linear transformation method is not easy to implement for some complex optimization problems in it. The PCA method outperforms the ordinary Brownian bridge method in most examples, so we use PCA method to conduct the highdimensional problem in the QMC. Furthermore, to get better convergence and reduce the actual error in QMC, we employ the RQMC method instead of QMC.

Based on the above analysis, this paper uses the Randomized Quasi-Monte Carlo (RQMC) method to simulate the paths of state variables. That means step 1 of the convertible bond pricing in Section 3.1 can be refined as the following steps.

(a) Generate $d$-dimensional Sobol sequence in $[0,1]^{d}$ denoted by

$$
\left\{x^{(i)}\right\}, \quad i=1,2, \ldots, M
$$

(b) Generate the stochastic variables with uniform distribution in $[0,1]^{d}$ denoted by $\varepsilon^{(i)}$. Let

$$
z^{(i)}=\bmod \left\{x^{(i)}+\varepsilon^{(i)}, 1\right\} .
$$

(c) Convert the $z^{(i)}$ into random numbers of normal distribution with Moro algorithm.

(d) Reduce the dimension with PCA method; then get the two-dimensional Brownian motion $\left(B_{t_{i}}^{1}, B_{t_{i}}^{2}\right)$.

(e) Obtain $M$ paths of each state variable with the corresponding difference equation.

\section{Numerical Experiment}

This section makes a numerical experiment for the convertible bond pricing in risk-neutral space and explores the influence of stochastic interest rate and credit risk on convertible bond prices. Firstly, with the stock price model of (5) and Ito's formula, we get

$$
\begin{aligned}
d \ln S_{t} & =\frac{1}{S_{t}} d S_{t}-\frac{1}{2} \frac{1}{S_{t}^{2}}\left(d S_{t}\right)^{2} \\
& =\left[r_{t}-\frac{1}{2}\left(\sigma_{1}^{2}+\sigma_{2}^{2}\right)\right] d t+\sigma_{1} d B_{t}^{1}+\sigma_{2} d B_{t}^{2}
\end{aligned}
$$

Then the stock price process satisfies the following difference equation:

$$
\begin{aligned}
S_{t+\Delta t}=S_{t} \times \exp \{ & {\left[r_{t}-\frac{1}{2}\left(\sigma_{1}^{2}+\sigma_{2}^{2}\right)\right] \Delta t } \\
& \left.+\sigma_{1}\left(B_{t+\Delta t}^{1}-B_{t}^{1}\right)+\sigma_{2}\left(B_{t+\Delta t}^{2}-B_{t}^{2}\right)\right\},
\end{aligned}
$$

and with the interest rate model of (4), the process of interest rate satisfies the following difference equation:

$$
r_{t+\Delta t}=r_{t}+\left(a-b r_{t}\right) \Delta t+\sqrt{c r_{t}+d}\left(B_{t+\Delta t}^{1}-B_{t}^{1}\right) .
$$

We consider a three-year convertible bond with call and put provisions. Assume that the face value of the convertible bond is 100 , the annual interest rate is $2.5 \%$, the interest is paid at the end of each year, and the initial conversion price is $p=28$; then the convertible value for time $t$ is $V_{t}^{\text {con }}=\left(S_{t} / P\right) \times 100$. Other parameters in the given model are as follows:

$$
\begin{array}{rr}
S_{0}=22, & r_{0}=0.06, \\
a=0.05, & b=0.012, \\
c=0.054, & d=0.036, \\
\sigma_{1}=0.08, & \sigma_{2}=0.11, \\
\Delta t=\frac{1}{100}, \\
V^{\text {call }}=120, \quad V^{\text {put }}=102, \\
\lambda=0.08, \quad \delta=0.5 .
\end{array}
$$

On Matlab software, we get the price of the convertible bond which is 97.86. Figure 1 shows the optimal exercise time of the convertible bond with call and put provisions. When there is no restrictive period to call and put provisions, the optimal exercise time of the convertible bond will centralize in the earlier stage. As a matter of fact, convertible bonds existing in the financial market have some banned puttable period.

\section{Empirical Analysis}

5.1. Data. In this section, we make an empirical study using the data of China's stock market. In recent years, the convertible bond market in China has developed rapidly. In 2012, the issuance of convertible bonds in China is nearly 100 billion Yuan. By the end of 2012, there are 21 convertible bonds traded in Shanghai stock exchange and Shenzhen stock exchange, 15 of which are in Shanghai stock exchange. The credit ratings of convertible bonds in China are all above AA-, and 10 of them are AAA, and only 2 are AA-. In China's market, the face value of all the convertible bonds is 100 Yuan, with maturities of generally 5-6 years, annual interest rates less than one-year bank deposit interest rate which is $3 \%$ now, and the interest paid once each year. Convertible bonds existing in China's market have four additional provisions, namely, general call provisions, conditional call provisions 


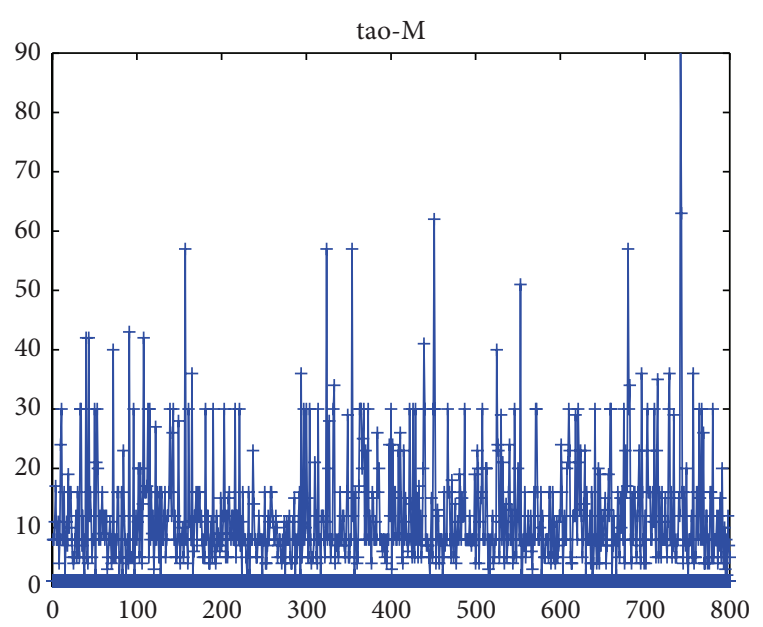

FIgURE 1: Optimal exercise time of convertible bond.

(soft call provisions), conditional put provisions, and general put provisions. Considering that the credit risk is involved in the pricing model of the convertible bond, we choose two convertible bonds, XGZZ and BHZZ, with different credit ratings as the sample, whose additional provisions both are the call provision and the general put provision.

The time interval of the sample data is from October 2009 to October 2012, the stock price is the daily closing price, and the interest rate model is estimated based on the closing yield to maturity of treasury. The data of convertible bonds prices, underlying stocks prices, and yield to maturity of treasury are taken from China Stock Market Accounting Research (CSMAR) database.

5.2. Stock Price and Interest Rate. Now, we estimate the parameters in stock price model and interest rate model with MLE method, and the likelihood function needs to be deduced firstly. We assume that the market price of risk equals 0 . With difference equation (28), we get

$$
\begin{aligned}
& \left(\ln S_{t+\Delta t} \mid \ln S_{t}\right) \\
& \quad \sim N\left(\ln S_{t}+\left[r_{t}-\frac{1}{2}\left(\sigma_{1}^{2}+\sigma_{2}^{2}\right)\right] \Delta t,\left(\sigma_{1}^{2}+\sigma_{2}^{2}\right) \Delta t\right) .
\end{aligned}
$$

With difference equation (29), one has

$$
\left(r_{t+\Delta t} \mid r_{t}\right) \sim N\left(r_{t}+\left(a-b r_{t}\right) \Delta t,\left(c r_{t}+d\right) \Delta t\right) .
$$

So the joint distribution of $\left(\ln S_{t+\Delta t}, r_{t+\Delta t} \mid \ln S_{t}, r_{t}\right)$ follows the bivariate normal distribution $N_{2}(\mu, \Sigma)$, where

$$
\begin{gathered}
\mu=\left(\begin{array}{c}
\ln S_{t}+\left[r_{t}-\frac{1}{2}\left(\sigma_{1}^{2}+\sigma_{2}^{2}\right)\right] \Delta t \\
r_{t}+\left(a-b r_{t}\right) \Delta t
\end{array}\right), \\
\Sigma=\left(\begin{array}{cc}
\left(\sigma_{1}^{2}+\sigma_{2}^{2}\right) \Delta t & \sigma_{1} \sqrt{c r_{t}+d} \Delta t \\
\sigma_{1} \sqrt{c r_{t}+d} \Delta t & \left(c r_{t}+d\right) \Delta t
\end{array}\right),
\end{gathered}
$$

and the correlation coefficient is $\rho=\sigma_{1} / \sqrt{\sigma_{1}^{2}+\sigma_{2}^{2}}$.
We divide the time interval $[0, T]$ into $n$ equal parts, then $\Delta t=T / n$, and the stock price and interest rate of the $k$ th part are $S_{k}$ and $r_{k}$. Based on the above analysis, we get the likelihood function as follows:

$$
\begin{aligned}
L\left(a, b, c, d, \sigma_{1}, \sigma_{2}\right) & =P\left(\ln S_{1}, \ln S_{2}, \ldots, \ln S_{n} ; r_{1}, r_{2}, \ldots, r_{n}\right) \\
& =\prod_{k=1}^{n-1} P\left(\ln S_{k+1}, r_{k+1} \mid \ln S_{k}, r_{k}\right),
\end{aligned}
$$

where $P\left(\ln S_{k+1}, r_{k+1} \mid \ln S_{k}, r_{k}\right)$ can be obtained with the joint density function of $\left(\ln S_{t+\Delta t}, r_{t+\Delta t} \mid \ln S_{t}, r_{t}\right)$. On Matlab software and with historical data, we get the above parameter estimates, which are denoted by $\widehat{a}, \widehat{b}, \widehat{c}, \widehat{d}, \widehat{\sigma}_{1}, \widehat{\sigma}_{2}$.

After obtaining the parameter estimates $\widehat{a}, \widehat{b}, \widehat{c}, \widehat{d}, \widehat{\sigma}_{1}, \widehat{\sigma}_{2}$, we can use the RQMC method to simulate the process of stock prices and interest rates with difference equations (28) and (29).

5.3. Default Rate and Recovery Value. For each path, we can obtain the optimal exercise time $\tau$ for convertible bonds with the LSROM method, and the interest rate of a risky bond is $r_{\tau}^{*}$ and the interest rate of risk-free bonds is $R_{\tau}$. Both of them can be obtained with the term structure of interest rate. The recovery rate is a constant of $\delta$ when the bond defaults; then the average default intensity at time interval $[0, \tau]$ is

$$
\lambda_{\tau}^{*}=\frac{1-e^{R_{\tau}-r_{\tau}^{*}}}{1-\delta} .
$$

So, the price of the convertible bond at time 0 can be written as

$$
\begin{aligned}
V_{0}= & \frac{1}{M} \sum_{i=1}^{M} e^{-r\left(\omega_{i} ; 0\right) \cdot \Delta t} \\
& \cdot\left[\prod_{t=t_{1}}^{t_{\tau}\left(\omega_{i}\right)}\left(1-\lambda_{t}\right) \cdot C\left(\omega_{i} ; t_{1}\right)\right. \\
& +\prod_{t=t_{1}}^{t^{*}\left(\omega_{i}\right)}\left(1-\lambda_{t}\right) D\left(\omega_{i} ; t_{\tau}\right)+\lambda_{t_{1}} \delta_{t_{1}} F \\
& +\sum_{n=1}^{\tau\left(\omega_{i}\right)-1} e^{-\sum_{m=1}^{n} r\left(\omega ; t_{m}\right) \cdot \Delta t} \\
& \left.\times\left(\prod_{m=1}^{n}\left(1-\lambda_{t_{m}}\right)\right) \lambda_{t_{n+1}} \delta F\right] .
\end{aligned}
$$

5.4. Basis Functions. Stentoft [27] made numerical analysis to the deviation of American option price which is obtained with the LSM method through choosing a different number of basis functions. The results show that there is no monotonous relationship between the number of basis functions and the pricing deviation. That is to say, increasing the number of basis functions does not always decrease the pricing 
TABLE 2: Sample of convertible bonds.

\begin{tabular}{lccccc}
\hline Item & Issuing date & Maturity date & Conversion price & Provision & Credit rating \\
\hline XGZZ & 2008.8 .21 & 2013.8 .21 & 5.88 Yuan & Call, put & AA+ \\
BHZZ & 2009.9 .23 & 2014.9 .23 & 6.16 Yuan & Call, put & AA- \\
\hline
\end{tabular}

TABLE 3: Estimates of parameters in convertible bond pricing model.

\begin{tabular}{lcccccc}
\hline Name & $\widehat{a}$ & $\widehat{b}$ & $\widehat{c}$ & $\widehat{d}$ & $\widehat{\sigma}_{1}$ & 0.2534 \\
\hline XGZZ & 0.0351 & 0.1264 & 0.0693 & 0.05694 & 0.1053 \\
BHZZ & 0.0492 & 0.0588 & 0.1042 & 0.1257 & 0.3291 & 0.0859 \\
\hline
\end{tabular}

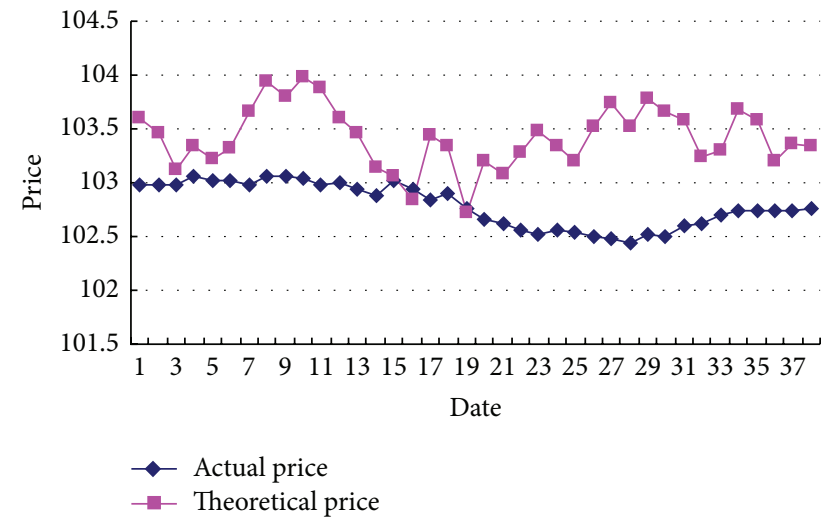

Figure 2: Comparison of theoretical price with actual price of XGZZ.

deviation, especially in the case of the out-of-the-money option. However, increasing the paths of simulation and basis functions will contribute to the pricing deviation reduction.

As to numerical stability, Laguerre polynomial is not the optimal choice for basis functions, but the converted Legendre polynomial is more effective than the general Chebyshev polynomial. Considering the calculation time and the computational accuracy, the better choice is the ordinary monomial for regressor. Since an increase in the number of basis functions cannot reduce the pricing deviation significantly but increase the computational amount greatly, we select ordinary monomials as basis functions with the number $K=5$, that is, $S_{t}, r_{t}, S_{t}^{2}, r_{t}^{2}, S_{t} r_{t}$, respectively.

5.5. Results. This paper selects the data from October, 2009, to September, 2012, as the sample and obtains the estimate parameters of the convertible bond pricing models with the MLE method as shown in Table 3. Furthermore, we obtain the comparison between theoretical prices and real market prices of the two convertible bonds, XGZZ and BHZZ, from October to November, 2012, as shown in Figures 2 and 3, respectively. The average pricing deviation of XGZZ is $2.72 \%$, and the average pricing deviation of $\mathrm{BHZZ}$ is $1.86 \%$. Obviously, the pricing of BHZZ is better than XGZZ's. The different deviations between the two convertible bonds may be due to the different terms to maturity; XGZZ is closer to the maturity date than BHZZ.

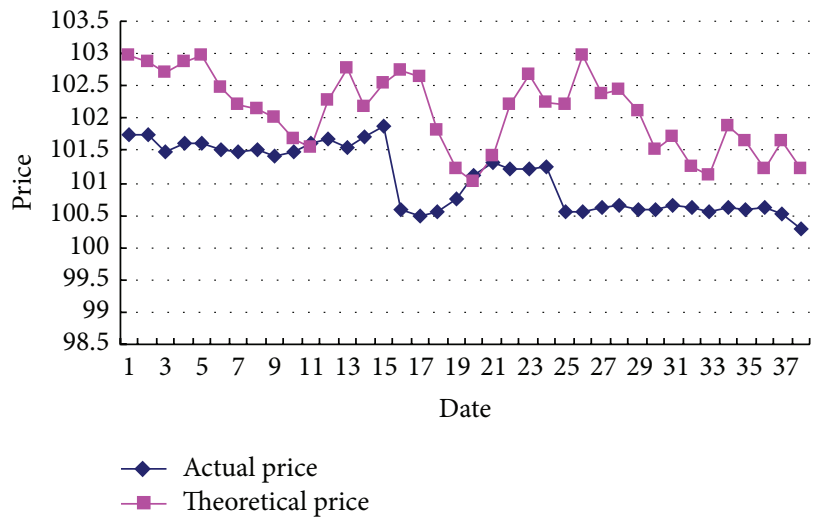

Figure 3: Comparison of theoretical price with actual price of BHZZ.

\section{Conclusions}

This paper studies the pricing problem of convertible bonds with LSRQM method. Extending existing approaches, the method is capable of accounting for the complex convertible bond with call and put provisions. Pricing convertible bonds with Randomized Quasi-Monte Carlo simulation is more flexible than previous Monte Carlo methods because it is effective for high-dimensional cases.

We model the financial market with stochastic interest rates and credit risk with suitable stochastic differential equations and describe the credit risk with Jarrow and Turnbull model. Furthermore, we present detailed calculation steps of convertible bonds values with LSRQM approach. Based on the theoretical analysis, we make a numerical simulation under determinate parameters and an empirical analysis using data in China's market. The results verify the validity of the proposed method.

\section{Conflict of Interests}

The authors declare that there is no conflict of interests regarding the publication of this paper.

\section{Acknowledgments}

The authors would like to express their gratitude to the supports given by the Natural Science Foundation of China 
(no. 71201013), the National Natural Science Innovation Research Group of China (no. 71221001), and the Innovation Platform Open Funds for Universities in Hunan Province of China (no. 13K059).

\section{References}

[1] J. C. Cox, S. A. Ross, and M. Rubinstein, "Option pricing: a simplified approach," Journal of Financial Economics, vol. 7, no. 3, pp. 229-263, 1979.

[2] M. Ammann, A. Kind, and C. Wilde, "Simulation-based pricing of convertible bonds," Journal of Empirical Finance, vol. 15, no. 2, pp. 310-331, 2008.

[3] F. A. Longstaff and E. S. Schwartz, "Valuing American options by simulation: a simple least-squares approach," Review of Financial Studies, vol. 14, no. 1, pp. 113-147, 2001.

[4] L. Stentoft, "Assessing the Least Squares Monte-Carlo approach to American option valuation," Review of Derivatives Research, vol. 7, no. 2, pp. 129-168, 2004.

[5] P. A. Acworth, M. Broadie, and P. Glasserman, "A comparison of some Monte Carlo and quasi Monte Carlo techniques for option pricing," in Monte Carlo and Quasi-Monte Carlo Methods 1996, pp. 1-18, Springer, New York, NY, USA, 1998.

[6] J. Imai and K. S. Tan, "Minimizing effective dimension using linear transformation," in Monte Carlo and Quasi-Monte Carlo Methods 2002, pp. 275-292, Springer, Berlin, Germany, 2004.

[7] W. J. Morokoff, "Generating quasi-random paths for stochastic processes," SIAM Review, vol. 40, no. 4, pp. 765-788, 1998.

[8] B. Moskowitz and R. E. Caflisch, "Smoothness and dimension reduction in quasi-Monte Carlo methods," Mathematical and Computer Modelling, vol. 23, no. 8-9, pp. 37-54, 1996.

[9] F. Wen, Z. He, and X. Chen, "Investors'risk preference characteristics and conditional skewness," Mathematical Problems in Engineering, vol. 2014, Article ID 814965, 14 pages, 2014.

[10] R. A. Jarrow and S. M. Turnbull, "Pricing derivatives on financial securities subject to credit risk," Journal of Finance, vol. 50, no. 1, pp. 53-85, 1995.

[11] M. W. Hung and J. Y. Wang, "Pricing convertible bonds subject to default risk," The Journal of Derivatives, no. 4, pp. 75-87, 2002.

[12] R. C. Donald and L. Qin, "A tree model for pricing convertible bonds with equity, interest rate, and default risk," The Journal of Derivatives, no. 2, pp. 25-46, 2007.

[13] J. Liu, J. Xiao, L. Yan, and F. Wen, "Valuing catastrophe bonds involving credit risks," Mathematical Problems in Engineering, vol. 2014, Article ID 563086, 6 pages, 2014.

[14] R. Xu, "A lattice approach for pricing convertible bond asset swaps with market risk and counterparty risk," Economic Modelling, vol. 28, no. 5, pp. 2143-2153, 2011.

[15] J. Liu, M. Tao, C. Ma, and F. Wen, "Utility indifference pricing of convertible bonds.", International Journal of Information Technology \& Decision Making, vol. 13, no. 2, pp. 429-444, 2014.

[16] R. Litterman and T. Iben, "Corporate bond valuation and the term structure of credit spreads," Journal of Portfolio Management, pp. 52-64, 1991.

[17] D. Duffie, “Credit swap valuation," Financial Analysts Journal, vol. 55, no. 1, pp. 73-87, 1999.

[18] L. Andersen and D. Buffum, "Calibration and implementation of convertible bond models," Journal of Computational Finance, vol. 7, no. 2, pp. 1-34, 2004.

[19] V. Linetsky, "Pricing equity derivatives subject to bankruptcy," Mathematical Finance, vol. 16, no. 2, pp. 255-282, 2006.
[20] P. Carr and V. Linetsky, "A jump to default extended CEV model: an application of Bessel processes," Finance and Stochastics, vol. 10, no. 3, pp. 303-330, 2006.

[21] D. Duffie, L. Saita, and K. Wang, "Multi-period corporate default prediction with stochastic covariates," Journal of Financial Economics, vol. 83, no. 3, pp. 635-665, 2007.

[22] J. Liu, L. Yan, and C. Ma, "Pricing options and convertible bonds based on an actuarial approach," Mathematical Problems in Engineering, vol. 2013, Article ID 676148, 9 pages, 2013.

[23] K. Tsiveriotis and C. Femandes, "Valuing convertible bonds with default risk," Journal of Fixed Income, vol. 8, no. 2, pp. 95102, 1998.

[24] C. Huang, C. Peng, X. Chen, and F. Wen, "Dynamics analysis of a class of delayed economic model," Abstract and Applied Analysis, vol. 2013, Article ID 962738, 12 pages, 2013.

[25] J. Lin and X. Wang, "New Brownian bridge construction in quasi-Monte Carlo methods for computational finance," Journal of Complexity, vol. 24, no. 2, pp. 109-133, 2008.

[26] L. Yu, S. Wang, F. Wen, and K. K. Lai, "Genetic algorithm-based multi-criteria project portfolio selection," Annals of Operations Research, vol. 197, pp. 71-86, 2012.

[27] L. Stentoft, "Assessing the least squares Monte-Carlo approach to American option valuation," Review of Derivatives Research, vol. 7, no. 2, pp. 129-168, 2004. 


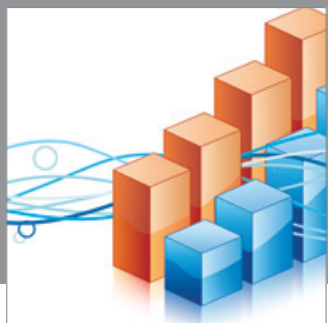

Advances in

Operations Research

mansans

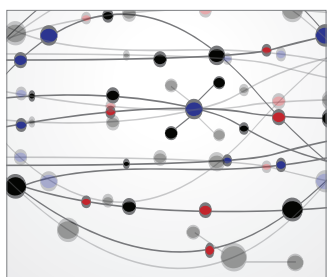

The Scientific World Journal
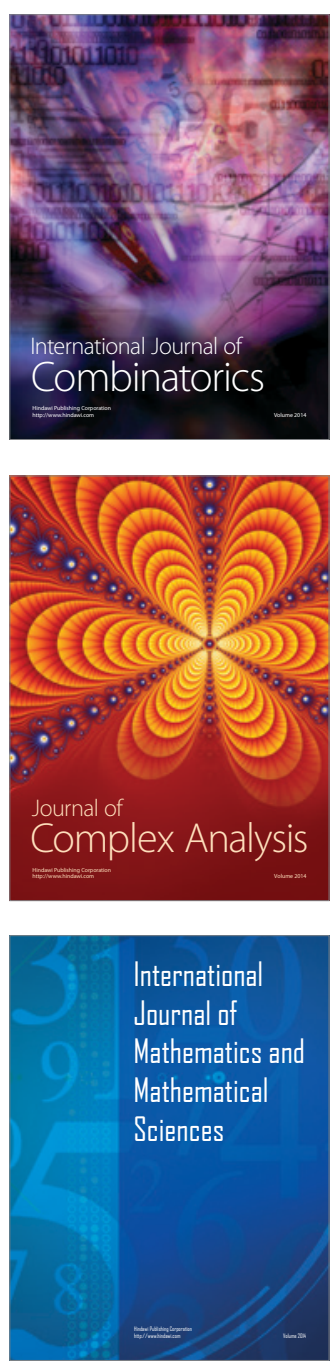
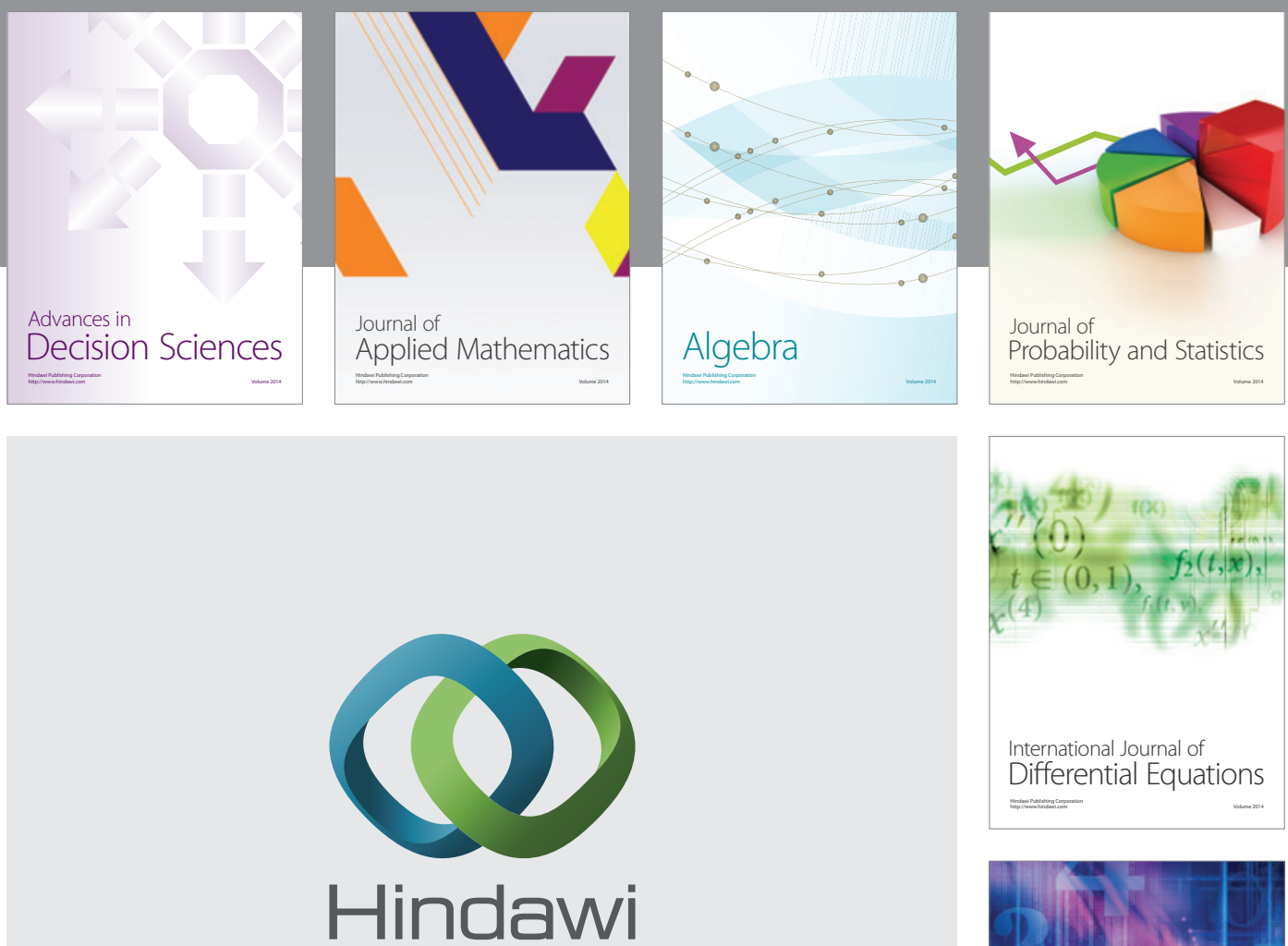

Submit your manuscripts at http://www.hindawi.com
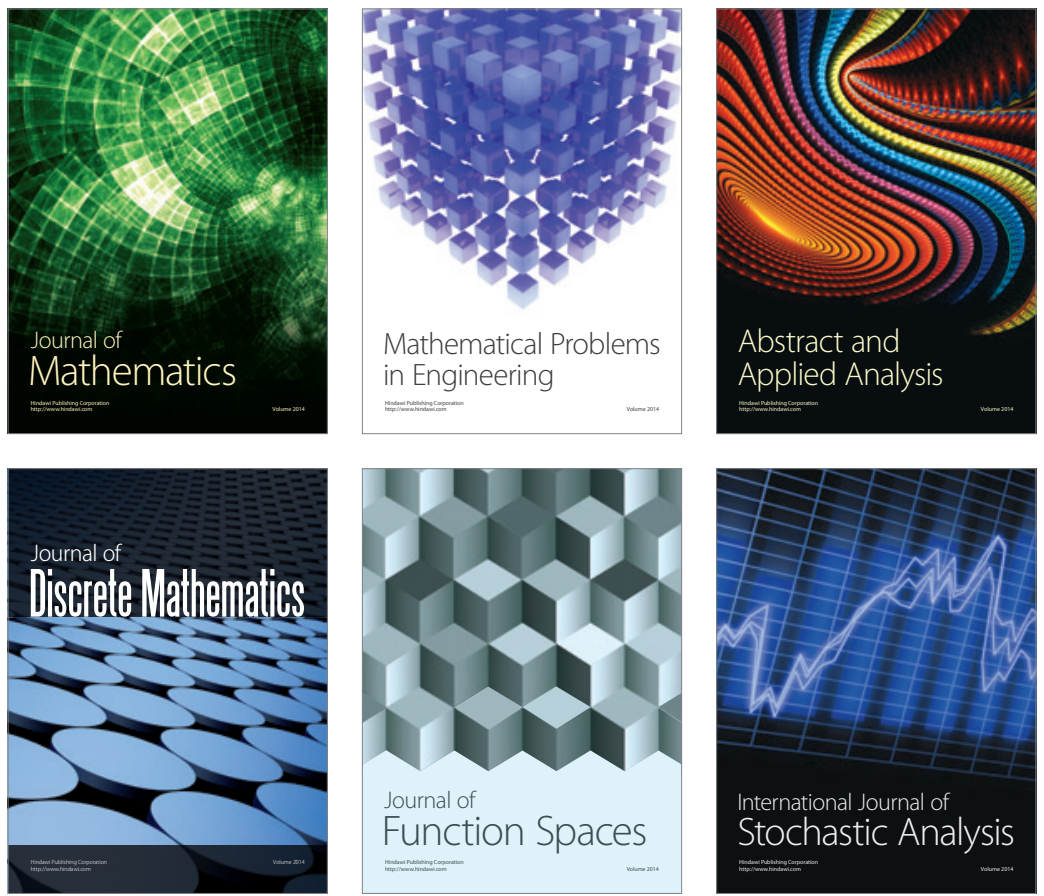

Journal of

Function Spaces

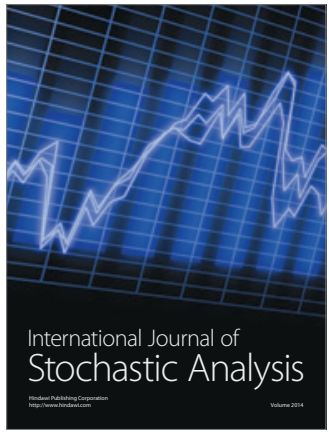

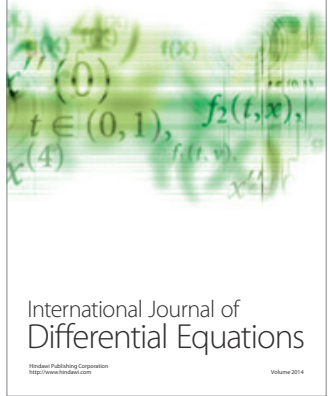
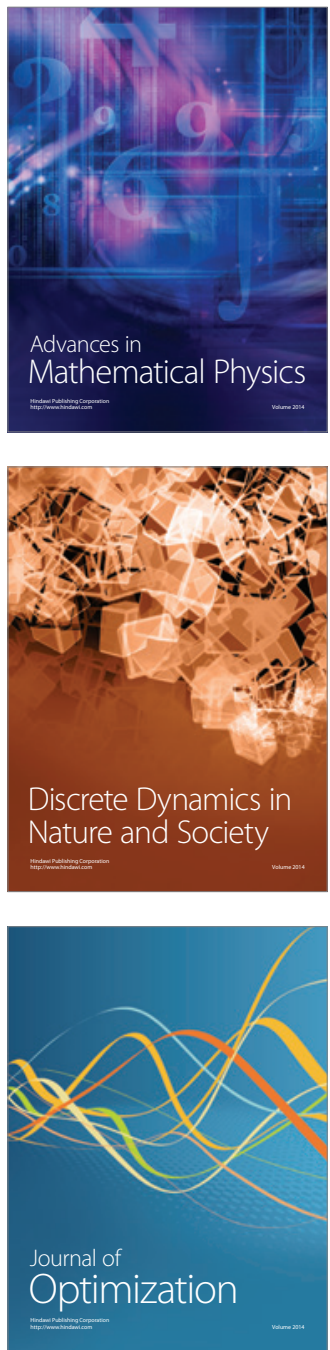